\title{
Application of Mid-Infrared Spectroscopy in the Quality Control of Traditional Chinese Medicines
}

Authors

Affiliations
Suqin Sun ${ }^{1}$, Jianbo Chen ${ }^{1}$, Qun Zhou ${ }^{1}$, Guanghua Lu ${ }^{2}$, Kelvin Chan ${ }^{3}$

The affiliations are listed at the end of the article
Key words

- traditional Chinese medicine

- quality control

- mid-infrared spectroscopy

- two-dimensional correlation infrared spectroscopy

- chemometric techniques $\begin{array}{ll}\text { received } & \text { February 11, } 2010 \\ \text { revised } & \text { Sept. 29, 2010 }\end{array}$

accepted October 7, 2010

\section{Bibliography}

Dol http://dx.doi.org/

10.1055/s-0030-1250520

Published online November 3 , 2010

Planta Med 2010; 76:

1987-1996 @ Georg Thieme

Verlag KG Stuttgart · New York .

ISSN 0032-0943

\section{Correspondence}

\section{Prof. Suqin Sun}

Department of Chemistry

Tsinghua University

Beijing 100084

China

Phone: + 861062787661

Fax: + 861062782485

sunsq@mail.chem.tsinghua.

edu.cn

Correspondence

Prof. Guanghua Lu

School of Pharmacy

Chengdu University

of Traditional Chinese Medicine

Chengdu 611137

China

Phone: + 862861800231

luguanghua@hotmail.com

\section{Abstract}

$\nabla$

Chinese herbal medicines are often referred to as Chinese materia medica (CMM). Composite formulae containing mixtures of CMM are prescribed for treatment and prevention of diseases in the practice of traditional Chinese medicine (TCM). Some of the well-known CMM formulae (Fufang in Chinese) are manufactured and marketed as proprietary Chinese medicines (PCM). Quality assessment and assurance of these products are difficult; they are a challenging task. Mid-infrared spectroscopy, a classic molecular structure analysis method, has been innovatively applied in the quality control of TCM, and has gained significant impact and advancement in analytical fields. Infrared fingerprinting features appear particularly suitable for the identification of multicomponent matrices in samples whose chemical integrity has not been altered or destroyed because no extraction procedure is needed. This review summarizes and gives an overall view on the application of mid-infrared and two-dimensional correlation infrared (2D-IR) spectroscopy as well as chemometric techniques in the identification of CMM, investigation of TCM processing procedures, and analysis of herb extracts and preparations.

\section{Introduction \\ $\nabla$}

Traditional Chinese medicines (TCM) include Chinese medicinal materials (CMM), CMM extracts, and proprietary Chinese medicines (PCM)/composite formulae, which contain complex chemical compositions. In general, pharmacological screenings and clinical tests show that multichemical compounds in TCM preparations are bioactive contributing to the overall therapeutic effect for that specific preparation. Similarly, for a holistic approach towards disease treatment in

\section{Abbreviations}

$\nabla$

2D-IR: two-dimensional correlation infrared

ANN: artificial neural network

ATR: attenuated total reflectance

CMM: $\quad$ Chinese materia medica

FSD: $\quad$ Fourier self-deconvolution

FT-IR: Fourier transform infrared spectroscopy

HCA: hierarchical clustering analysis

IRMFA: infrared spectroscopic macro-fingerprints analysis

M-IR: mid-infrared spectroscopy

NNM: nearest neighbor method

PCA: principal component analysis

PCM: $\quad$ proprietary Chinese medicines

PLS: $\quad$ partial least square

RBF: radial basis function

SD-IR: second derivative infrared

SIMCA: soft independent modeling of class analogies

SVM: $\quad$ support vector machine

TCM: traditional Chinese medicine

TIRIA: tri-level infrared spectroscopic identification analysis individual patients, the TCM doctor prescribes selected CMM in the form of herbal decoctions. This is essentially how CMM are used for prevention and treatment of diseases. It is difficult to identify a single chemical marker or the most representative marker contributing to the medicine function of a CMM. Thus, the quality of TCM products still remains a problem to be effectively evaluated, controlled, and assured. Over the past decade two directions on the quality control of TCM have been reported in the literature and adopted by the Pharmacopoeia using different chemometric 
and chromatographic analyses. One involves the qualitative and quantitative assay of one or several chemical markers, the other utilizes the fingerprinting technique. Because of the complex compositions of CMM and multi-herb PCM, it is generally accepted in the academic circle that fingerprinting is the most common technique used in the quality control of TCM products with the aid of analytical techniques such as chromatography, electrophoresis, or spectroscopy pattern-recognition reported in research publications [1]. Mid-infrared spectroscopy has been innovatively employed to identify and assess the quality of TCM products. The objective of this review is to summarize the application of mid-infrared techniques in the quality control of TCM products.

The mid-infrared spectroscopy $\left(4000-400 \mathrm{~cm}^{-1}\right)$ is one of the traditional spectroscopic methods to elucidate the molecular structure of an unknown chemical compound. Infrared fingerprints can provide some information of molecular structure by comparing the infrared spectra of an unknown sample with an authentic sample. Thus, mid-infrared spectroscopy is a conventional method to control the quality of many pharmaceutical drugs. In measuring the spectra of complex mixtures, such as cells, tissues, food, and TCM, mid-infrared spectrum provides an overlapped fingerprint of all chemical compositions in the tested samples. Minute changes in tested samples might be detected by the variations of fingerprints. This is because in screening fingerprints, a modern Fourier transform infrared spectrometer with the high ratio of signal-to-noise monitored by various sampling techniques (e.g., attenuated total reflectance [ATR] accessory, various analytical techniques by computer software, etc.) can be used for data-analysis.

The advantages of mid-infrared techniques employed in the quality control of TCM are found on sample preparations [2-5]. TCM samples can be directly and rapidly tested to obtain an infrared spectrum, because they are not extracted or separated and the preparation procedure is nondestructive. The infrared spectrum fingerprint shows the "whole" chemical information of all chemical compositions in the TCM sample, which is consistent with the philosophy of the traditional principles of TCM. Combining evaluation methods of infrared spectral data, some chemical compounds can be qualitatively and quantitatively analyzed. However, difficulty of maintaining test samples water-free and lack of promotion of this technique are the limitations of the mid-infrared spectroscopy. Nevertheless, many publications have concluded that the mid-infrared technique is suitable for identification of TCM herbs, investigation of TCM processing, and quality control of the TCM preparations.

\section{Theoretical Principles and Analytical Procedure of Mid-Infrared Spectroscopy \\ $\nabla$}

The mid-infrared spectrum is considered as the overlapped spectrum of all chemical compositions. The infrared spectral peaks for a particular function group in the molecular structure are located at the same spectral region. The information for a class of chemical compounds with similar molecular structures can be deduced. For example, the peak at $1745 \mathrm{~cm}^{-1}$ is assigned to the stretch vibration of $\mathrm{C}=\mathrm{O}$ bonds in pure glycerin tripalmitate. If a peak is located at this position of the infrared spectrum, it may indicate that this herbal sample contains ester compounds and related groups. Therefore, chemical information about TCM samples can be obtained by comparing the positions of overlapped peaks to those of authenticated TCM reference samples or chemical standards. With the help of multivariate calibration models, some chemical compounds can be quantified in TCM samples. The derived pattern of IR spectrum of a TCM sample can be considered as a spectroscopic fingerprint for this specific sample. This fingerprint is defined as "macro-fingerprint" to be differentiated from the features of the pure compounds. The changes in peak position and intensity of the spectra can be related to the changing variety of chemical compositions in the sample. Hence, various TCM samples might be differentiated by their infrared spectra although their chemical compositions have not been exactly revealed or identified. Based on this principle, the true or fake herbs, good (defined upon the fact that their qualities have been proved in practice for thousands of years) or bad quality samples might be identified or referred to by their infrared spectra. The CMM originated from various species of plants or animals, geographical areas, and cultivating procedures which may differ in their chemical compositions and thus pharmacological effects. Distinguishing these samples is necessary and important to assure the quality and therapeutic effects of the TCM products used according to the Chinese medicine treatment theory. These variations in samples may be differentiated by infrared spectra. However, if the differences among infrared spectra of various TCM samples are too small to be observed, pattern recognition techniques are used to improve spectral resolution. The refinement techniques include hierarchical clustering analysis (HCA), principal component analysis (PCA), soft independent modeling of class analogies (SIMCA), artificial neural network (ANN), and support vector machine (SVM).

In the literature, tri-level infrared spectroscopic identification analysis (TIRIA) is used to identify CMM [6]. CMM samples can initially be identified and differentiated by comparing their FT-IR spectra, known as the primary identification. The secondary identification analysis is based on second derivative infrared (SD-IR) spectroscopy with a greater resolution than the primary infrared spectrum. The resolution can differentiate and separate some overlapped peaks on the primary infrared spectra resulting in better SD-IR spectra to distinguish the CMM samples. In the case that differentiation using infrared and SD-IR spectra is not possible for some samples, two-dimensional correlation infrared (2D-IR) spectroscopy can be used; which is known as tertiary identification analysis. 2D-IR or two-dimensional correlation spectroscopy was originally introduced and expanded by Noda [7-9] and provides a 3D-plot of the spectrum. The 2D-IR plots of the CMM samples can then be used to tell the differences apart.

\section{Identification of Chemical Compositions in TCM Samples \\ $\nabla$}

Mid-infrared spectra of TCM samples can provide some information of the molecular structures of chemical compositions. For example, herbal samples rich in vegetable fat such as Sinapis Semen (the seed of Sinapis alba L.), Cannabis Semen (the seed of Cannabis sativa L.), Raphani Semen (the seed of Raphanus sativus L.), and Mume Fructus (the nearly ripe fruit of Prunus mume [Sieb.] Sieb. et Zucc.) show strong absorption peaks at 2925, 2855, and $1745 \mathrm{~cm}^{-1}$, which are assigned to the anti- and symmetric stretch vibration of $\mathrm{C}-\mathrm{H}$ bonds of methylenes and the stretch vibration of $\mathrm{C}=\mathrm{O}$ bonds [5]. The high amount of proteins in the TCM samples originated from animal parts, e.g., Cervi Pantotrichum Cornu (the horn of the male beast of Cervus nippon Temminck), Saigae Tata- 
ricae Cornu (the horn of Saiga tatarica L.), Scorpio (the whole body of Buthus martensii Karsch), and Hirudo (the whole body of Hirudo nipponica Whitman) can be visualized as bands of amide I and II on their infrared spectra. The fact that Cervi Pantotrichum Cornu contains inorganic salt $\mathrm{Ca}_{3}\left(\mathrm{PO}_{4}\right)_{2}$ and Scorpio sulfates could be verified from their infrared spectra [10].

Coptidis Rhizoma (the rhizome of Coptis chinensis Franch.) contains a high level of berberine as visualized in the fingerprinting peaks found both on its infrared and SD-IR spectra, with the pattern being more obviously shown in the latter than in the former spectrum ( $\boldsymbol{O}$ Fig. 1 ). We also observed that the intensities of characteristic peaks changed with the varying amount of berberine in tested samples, which was in agreement with the result of the HPLC analysis [11].

Pei and coworkers analyzed Epimedii Herba (the branch and leaf of Epimedium brevicornu Maxim.) by infrared and HPLC methods. They figured out that the peak at $\sim 1259 \mathrm{~cm}^{-1}$ on its infrared spectrum was related to the $4^{\prime}$-methoxyl-prenylflavonols, which were considered as the main bioactive compounds in this herb [12]. Cheung et al. also identified this characteristic peak by wavelet analysis and radial basis function (RBF) using neural network [13]. Therefore, the absorption peak at $\sim 1259 \mathrm{~cm}^{-1}$ on the infrared spectrum may be used as a characteristic peak to rapidly and effectively assess the quality of Epimedii Herba.

\section{Differentiation of Genuine and Fake TCM Herbs \\ $\nabla$}

Genuine and fake TCM herbs can be identified by infrared macrofingerprinting because the fake herbs must contain different chemical compositions compared to the true ones. Cao et al. distinguished the authentic Gastrodiae Rhizoma (the tuber of Gastrodia elata Bl.) from its counterfeit (the rhizome of Canna edulis Ker) by 2D-IR spectroscopy [14]. Although their infrared spectra were found to be similar, their 2D-IR spectra were significantly different. There were two strong auto-peaks located at 1237 and $1415 \mathrm{~cm}^{-1}$ in the range of $1500-800 \mathrm{~cm}^{-1}$ on synchronous 2D-IR spectrum of the genuine Gastrodiae Rhizoma, whilst the autopeaks in the counterfeit samples appeared at 1024, 1055, 1194, and $1225 \mathrm{~cm}^{-1}$. Zhou et al. [15] identified the authentic Rhei Radix et Rhizoma (the root and rhizome of Rheum tanguticum L.) and its fake one (the rhizome of Rheum franzenbachii Munt.) by infrared and 2D-IR spectra with thermal perturbation. The peak position and intensity on infrared spectra of these pair of herbs were very similar, but their $2 \mathrm{D}-\mathrm{IR}$ spectra were drastically different. In the region of $1700-1000 \mathrm{~cm}^{-1}$, only two auto-peaks located at 1460 and $1080 \mathrm{~cm}^{-1}$ occurred in the fake herb, whilst two additional auto-peaks occurred at 1560 and $1060 \mathrm{~cm}^{-1}$ in the genuine herb.

TIRIA is often utilized to identify genuine or fake TCM herbs. Sun et al. differentiated the genuine Pinellia Rhizoma (the rhizome of Pinellia ternata [Thunb.] Breit.) from its counterfeit with this technique [16]. In addition, the authentic and fake herbs, namely Pinellia Rhizoma [16], Asini Corii Colla (donkey hide stewed and concentrated as gelatinous mass, Equus asinus L.) [17] (○ Fig. 2), Glycyrrhizae Radix et Rhizoma (the root and rhizome of Glycyrrhiza uralensis Fisch.) [18], Anisi Stellati Fructus (the fruit of Illicium verum Hook. f.) [19], Codonopsis Radix (the root of Codonopsis pilosula [Franch.] Nannf.) [20], Rosae Rugosae Flos (the flower bud of Rosa rugosa Thunb.) [21], Cistanches Herba (the fleshy stem of Cistanche deserticola Y.C. Ma) [22] and Cordyceps (the stroma formed Cordyceps sinensis [Berk.] Sacc., a parasite of the
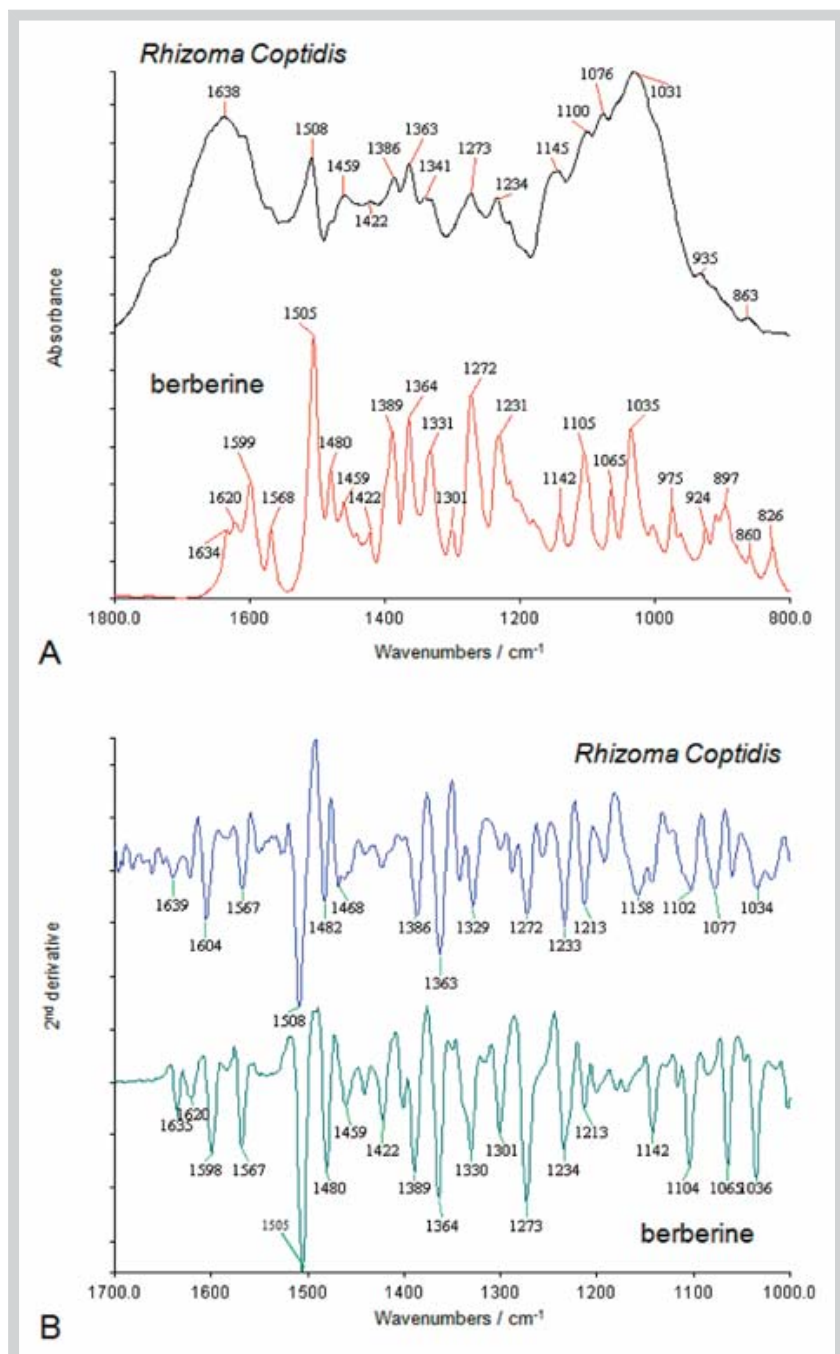

Fig. 1 Infrared (A) and second derivative infrared (B) spectra of Coptidis Rhizoma (the rhizome of Coptis chinensis Franch.) and berberine.
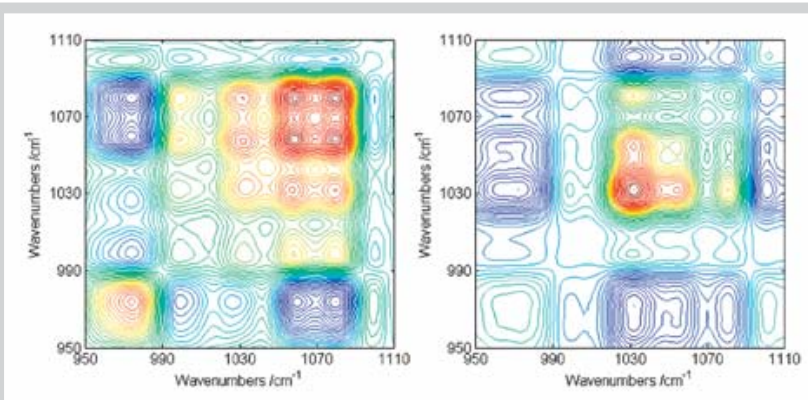

Fig. 2 2D-IR spectra of genuine (left) and false (right) herbs of Asini Corii Colla (donkey hide stewed and concentrated as gelatinous mass of Equus asinus L.).

larva of Hepialus armoricanus Oberthru.) [23] were successfully identified by this method.

The derivative infrared spectra and the Fourier self-deconvolution (FSD) method can separate overlapped peaks and enhance the resolution of the spectra during analysis. Cheng et al. differ- 
entiated genuine Gastrodiae Rhizoma samples from their counterfeits by the FSD-IR spectra [24]. The genuine and fake Corydalis Rhizoma (the tuber of Corydalis turtschaninovii Bess. f. yanhusuo Y.H. Chou et C.C. Hsu) [25] and Ophiopogonis Radix (the root tuber of Ophiopogon japonicus [Thunb.] Ker-Gawl.) [26] were differentiated by combining the derivative infrared spectra and statistic test methods. RBF neural network was also used to identify the genuine and fake Atractylodis Macrocephalae Rhizoma (the rhizome of Atractylodes macrocephala Koidz) [27] and Rhei Radix et Rhizoma [28] on the basis of infrared spectra.

\section{Differentiation of Chinese Herbs Collected from Different Geographical Regions \\ $\nabla$}

The proper and successful practice of Chinese medicine depends on the availability of good quality CMM samples, which should originate from their original cultivation areas. It is generally accepted that CMM originating from these areas are of the best quality. These CMM are referred to as "trueborn" "Daodi" in Chinese transliteration) from the original cultivation area. Those not grown in their geographical origins are considered as "non-trueborn" CMM. Samples from these different sources may result in various therapeutic effects. Prices between trueborn and nontrueborn samples are usually different in herbal markets. Infrared techniques were used to differentiate these kinds of samples based on the variation in their chemical compositions.

Han and coworkers [29] analyzed Puerariae Lobatae Radix (the root of Pueraria lobata [Willd.] Ohwi) samples collected from three different regions (Tianjin, Hunan, and Chongqing) in China by infrared and 2D-IR spectroscopy. All samples showed similar infrared spectra identified as starch but different intensities of the characteristic peaks characterized as puerarin. The samples collected from Tianjin showed stronger intensity than those of other regions, and their infrared spectra differed most from the starch. Similar observations were obtained from the SD-IR spectra. These results indicated that the quality of the samples collected from Tianjin might be better than the others. Other investigations using infrared and 2D-IR on CMM collected from different geographical areas were reported, e.g., Fritillariae Bulbus [30], Panacis Quinquefolii Radix (the root of Panax quinquefolium L.) [31], and Citri Reticulatae Pericarpium (the pericarp of Citrus reticulata Blanco) [32].

Some statistic classification methods are feasible to enhance the resolution of the infrared spectra for large numbers of samples. For the identification of trueborn and non-trueborn samples of Dioscoreae Rhizoma (the rhizome of Dioscorea opposita Thunb.), three different classification methods were applied. Sun et al. differentiated, with the aid of standard samples, trueborn from nontrueborn samples of Dioscoreae Rhizoma using the correlation coefficients of infrared spectra [33]. The correlation coefficients among the spectra of trueborn samples to those of standard samples were greater than 0.98 , whereas those of the non-trueborn samples were smaller than 0.98 . Xu and coworkers differentiated the samples of Dioscoreae Rhizoma collected from different cultivation areas by PCA analysis of FT-IR spectra. The scores of the samples on the second and third principal components were effective to differentiate the trueborn samples from non-trueborn ones [34]. The SIMCA classification method was also applied to differentiate the trueborn samples of Dioscoreae Rhizoma from the others [35]. Zhou and coworkers also used the SIMCA method to identify the samples of Lycii Fructus (the fruit of Lycium bar- barum L.) collected from three different regions [36]. Liu et al. [37] differentiated samples of Angelicae Dahuricae Radix (the root of Angelia dahurica Fisch. ex Hoffm.) and Salviae Miltiorrhizae Radix et Rhizoma (the root and rhizome of Salvia miltiorrhiza Bunge) collected from different cultivation regions by the nearest neighbor method (NNM) and a SVM-based multiclass classifier. The leave-one-out cross-validation accuracy of the NNM method was more than $96 \%$, whilst that of the SVM method was more than $99 \%$ for either of the two TCM herbs.

The PCA analysis of the infrared spectra of Scutellariae Radix (the root of Scutellaria baicalensis Georgi) samples collected from 15 administrative districts gave some interesting results [38]. All samples were separated into 6 groups by the first three principal components. Each of the groups was corresponded to several administrative districts with the same environment, climate, and geography conditions. A subsequent analysis by RBF neural network validated the classification results. The new result was more reasonable than the former one only when the actual administrative division was analyzed. Similar results occurred by PCA and RBF neural network analysis on the infrared spectra of 92 Paeoniae Rubra Radix (the root of Paeonia lactiflora Pall.) samples collected from 18 administrative districts [39].

\section{Identification of Wild and Cultivated Chinese Herbs $\nabla$}

The growing environment differences between cultivated and wild plants and the various cultivation procedures may result in a variation of chemical composition in the herbs. Their therapeutic effects are likely to be diverse. Hence, we also embarked on the identification of wild and cultivated samples using similar approaches.

Wang and coworkers [40] distinguished the wild and cultivated Salviae Miltiorrhizae Radix et Rhizoma by the TIRIA method. The infrared spectral peaks were located at 1050, 1144, and $1635 \mathrm{~cm}^{-1}$ in the cultivated samples, whilst the peaks were located at 1036 , 1155 , and $1623 \mathrm{~cm}^{-1}$ in the wild samples. On their SD-IR spectra, a single peak was located at $1410 \mathrm{~cm}^{-1}$ in the cultivated sample, while the wild sample had two peaks located at 1406 and $1420 \mathrm{~cm}^{-1}$. Instead of the peaks at 993 and $872 \mathrm{~cm}^{-1}$ on the SD-IR spectra in the cultivated sample, there was a peak at $1032 \mathrm{~cm}^{-1}$ in the wild samples. In the region of $1170-860 \mathrm{~cm}^{-1}$ on synchronous 2D-IR spectra, there were auto-peaks at 905, 970, 1011, 1100, and $1133 \mathrm{~cm}^{-1}$ in the cultivated, whilst the auto-peaks in the wild sample appeared at 908,950,973, 1068, 1099, and $1139 \mathrm{~cm}^{-1}$ (๑ Fig. 3). Liu et al. differentiated cultivated samples from wild ones of Ginseng Radix et Rhizoma (the root with rhizome of Panax ginseng C.A. Mey.) by the TIRIA method [41]. The wild and cultivated samples of Gastrodiae Rhizoma could be identified by infrared spectra [42].

Dong and coworkers discriminated cultivated from wild Paeoniae Rubra Radix by infrared spectra and SIMCA method. The recognition rate for the cultivated sample and rejection rates for both the wild and cultivated samples were $100 \%$. However, the recognition rate for the wild sample was only $83 \%$, which was considered to be due to the variety of growing regions. Nineteen other samples were used as an independent validation set to verify the performance of the SIMCA model. Seventeen of them were classified correctly [43-44]. The SIMCA method was also used in the differentiation of cultivated from wild Cistanches Herba. Both the recognition and rejection rates for the two classes were more than $90 \%$ [45]. Xu et al. differentiated the cultivated from the wild 

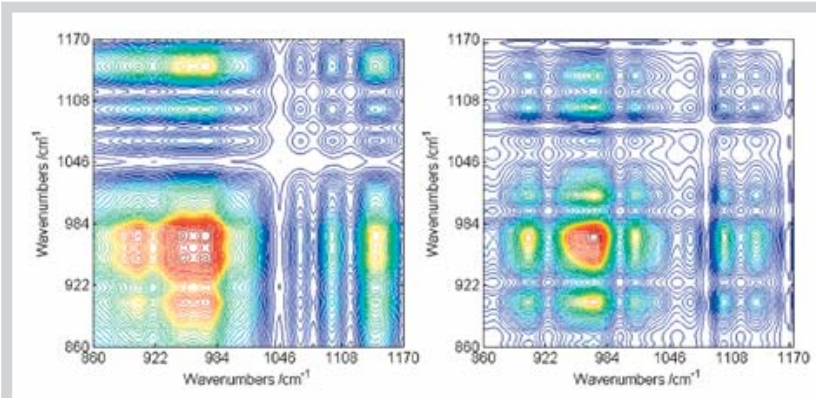

Fig. 3 2D-IR spectra of wild (left) and cultivated (right) Salviae Miltiorrhizae Radix et Rhizoma (the root and rhizome of Salvia miltiorrhiza Bunge).

sample of Scutellariae Radix by three kinds of BP-ANN methods. The recognition rate for the best model was more than $97 \%$ [46].

\section{Identification of Different Species of Chinese Herbs \\ $\nabla$}

Huang and coworkers [47] analyzed some typical herbal samples belonging to different families, such as Araliaceae, Campanulaceae, Magnoliaceae, Lauraceae, Leguminosae, Berberidaceae, and Cruciferae. The similarities and differences among the herbal samples in a specific family were also analyzed. The results indicated that the FT-IR technique was an effective method for the chemotaxonomy, which would be a supplement of the morphologic taxonomy.

The infrared spectra of Ginseng Radix et Rhizoma, Panacis Quinquefolii Radix and Notoginseng Radix et Rhizoma (the root of Panax notoginseng [Burk.] F.H. Chen) were much similar for the same matrix compositions. But the three groups of herbal samples were differentiated by either the SIMCA method or the SD-IR and 2D-IR spectra [48]. Wang et al. identified samples of Cimicifugae Rhizoma (the rhizome of Cimicifuga spp.) from 15 species of plants by infrared spectra. The differences between samples of different families were quite obvious [49]. The samples of Lycii Fructus (Gouqizi in Chinese transliteration) from 10 species of plants were identified by infrared spectra [50]. Pei et al. identified samples of Epimedii Herba from 5 species of plants by infrared and SD-IR spectra [51].

For the identification works using the TIRIA method, the abovementioned examples are normally compared using number, position, and approximate intensity of auto- and cross peak of the 2D-IR spectra. However, Chen et al. [52] introduced the quantitative analysis method by 2D-IR spectra and discriminated samples of Astragali Radix (Huangqi in Chinese transliteration) coming from different genera by the symmetry analysis of hetero 2D-IR spectra (O Fig. 4) and statistical test methods [53].

\section{Differentiation of CMM in Various Parts, Storage Duration, and Morphological Features $\nabla$}

Lu and coworkers differentiated the main root from the rootlets of Angelicae Sinensis Radix (the root of Angelica sinensis (Oliv.) Diels) by infrared and 2D-IR spectra [54]. Different spectra between the main root and the rootlets of the same plant indicated the inhomogeneous distribution of amino acids, essential oil, and sugar. Jin et al. [55] identified the root, stem, and leaf of Acantho-

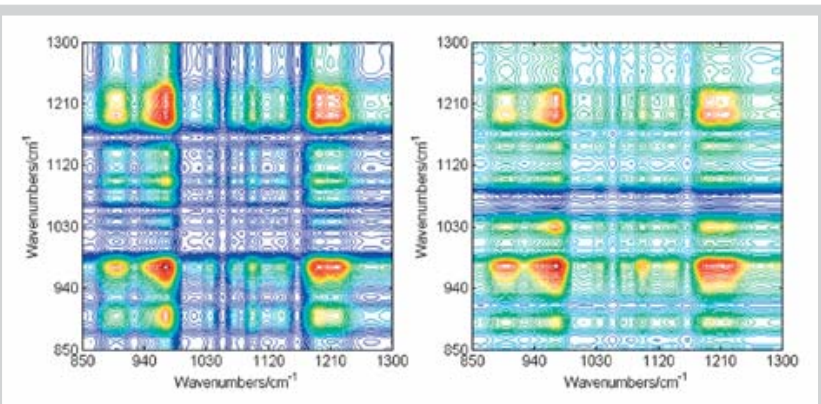

Fig. 4 Hetero 2D-IR spectra of Astragalus Radix (Huangqi in Chinese transliteration) samples belonging to plants of the same genus (Zhengheiqi, left) and of different genera (Huangqi, right).

panax senticosus (Rupr. et Maxim.) Harms by infrared and 2D-IR spectra. It was found that starch and calcium oxalate were abundant in the root and stem, whilst the leaves contained much more flavones than the other two plant parts. Xu et al. analyzed different parts of the stem of Cistanche deserticola Y.C. Ma by infrared and 2D-IR spectra and revealed that the chemical compositions were different in the cortex and core of this stem [56]. Hong et al. found that peoniflorin in the xylem of Paeoniae Alba Radix (the root of Paeonia lactiflora Pall.) was more abundant than that in the cortex by infrared spectra [57].

Zhan and coworkers applied wavelet transform to improve the resolution of 2D-IR spectra and successfully differentiated the various age samples of Ginseng Radix et Rhizoma [58]. During storage of Citri Reticulatae Pericarpium samples, the peak intensities at 2851,1716 , and $1516 \mathrm{~cm}^{-1}$ on the FT-IR spectra of its extract were increased, and peak positions were changed to 1734 , 1517 , and $1276 \mathrm{~cm}^{-1}$, which resulted from the increased amount of hesperidin, organic acids, and esters. The results reflected the fact that "the longer the storage duration of the Citri Reticulatae Pericarpium, the better quality of the herb" [59]. Moreover, Sun et al. successfully differentiated samples of Lycii Fructus in a variety of colors, shapes, tastes, and water content by FT-IR spectra [60]. Liu et al. analyzed the samples of Paeoniae Alba Radix collected from the Good Agricultural Practice base, herb markets, and purchased standard herbs [61].

\section{Quality Assessment of CMM during Processing \\ $\nabla$}

Some CMM must be processed by physical and/or chemical procedures before clinical use in order to decrease the side effects or improve therapeutic effects. It is valuable to reveal the fundamental physical and chemical processing to effectively control the quality of the processed sample and differentiate it from raw materials.

Yu and coworkers [62] investigated the processing of Rehmanniae Radix (the root of Rehmannia glutinosa Libosch, raw material) by yellow wine to produce Rehmanniae Radix Praeparata (processed sample) by infrared and 2D-IR spectra. Based on the changes of the infrared ( $\odot$ Fig. 5A), SD-IR ( $\odot$ Fig. 5B), and 2D-IR spectra of the samples stewed in yellow wine for various durations, it was revealed that stachyose was hydrolyzed into galactose, glucose, and fructose during herb processing. The mixture of glucose and fructose in the processed sample gave an overlap peak at ca. $777 \mathrm{~cm}^{-1}$, which was different from the peak at $771 \mathrm{~cm}^{-1}$ in raw materials. 

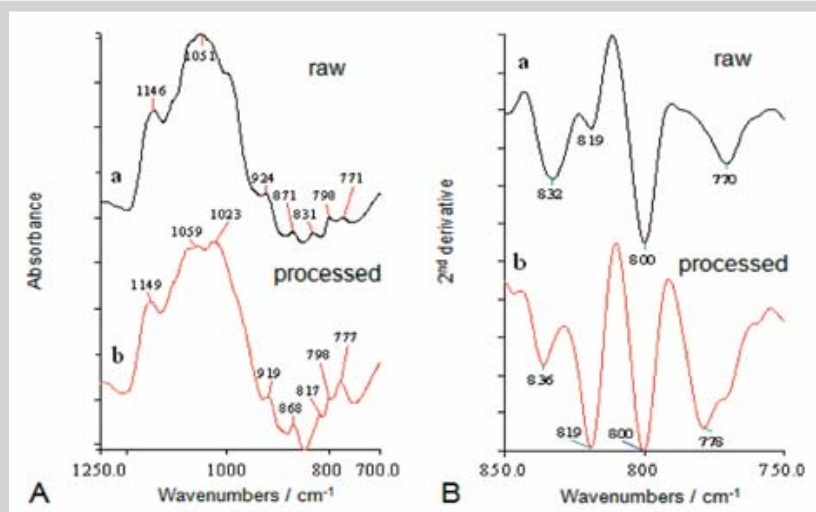

Fig. 5 Infrared (A) and second derivative infrared (B) spectra of Rehmanniae Radix (the root of Rehmannia glutinosa Libosch, raw material) and Rehmanniae Radix Praeparata (processed sample).

Meanwhile, melanoidin was produced by the chemical reaction between amino acids and monosaccharides. Hence, the processed sample appeared blacker in color. These results explained the reason why regular processed samples (Rehmanniae Radix Praeparata) should be sweet and appear black in color. It is possible that the processing procedure can be monitored and controlled by infrared techniques.

The processing procedure of Sinapis Semen was also studied using infrared and 2D-IR spectra [63]. The decreasing of amide I and II bands at about 1657 and $1546 \mathrm{~cm}^{-1}$, respectively, during herb processing indicated the loss of the proteins, which was consistent with the conventional processing principles. The absorption peak of cellulose at $\sim 1055 \mathrm{~cm}^{-1}$ was significantly decreased after processing for $10 \mathrm{~min}$ resulting in herbal samples turning yellow in color.

The raw and processed Aconiti Radix (the axial root of Aconitum carmichaeli Debx.) [64] and Aconiti Kusnezoffii Radix (the root of Aconitum kusnezoffii Reichb.) [65] were differentiated by infrared and 2D-IR spectra, as well as the Aconiti Lateralis Radix Praeparata processed in three different ways [66]. Bao et al. studied the effects of Chrysanthemi Flos (the capitulum of Chrysanthemum morifolium Ramat.) processing on the infrared spectra and found that the existence of the peak at $1714 \mathrm{~cm}^{-1}$ could be chosen as a marker to control the processing procedure [67]. Xu et al. investigated the changes of chemical compositions in Viticis Fructus (the fruit of $\mathrm{Vi}$ tex trifolia L. var. simplicifolia Cham.) by infrared and 2D-IR spectra during the processing procedures and further successfully differentiated the patterns of various processed samples [68].

\section{Quality Control of Herbal Extracts and Formula Granules \\ $\nabla$}

Extracting CMM in water or other solvents can eliminate unwanted constituents such as cellulose and starch, resulting in a herbal extract with high content of bioactive components. The herbal extract is further processed to herbal preparations such as granules of individual CMM or, if CMM composite formula (mixture of several CMM) is involved, CMM formula granules, CMM injection preparations, and other dosage forms. Liu and coworkers studied the extracts of Angelicae Sinensis Radix extracted by different procedures and observed that a high content of Z-ligustilide was found in the extracts of petroleum ether and water distillation, but the divergence of infrared, SD-IR, and 2D-IR spectra among these extracts was significantly different [69-70]. Extracts of Chrysanthemi Flos collected from seven cultivation regions using different solvents were analyzed by infrared and 2D-IR spectra [71]. The compositions in these extracts were found to vary with the geographical origins and extracting solvents. Wu et al. analyzed the water and alcohol extracts of Coptidis Rhizoma by infrared spectra and found that the amount of berberine in these extracts was greater than that in raw materials [72]. Formula granules are a new type of TCM preparation. They are usually manufactured from CMM using mixtures of solvent-free extracts of CMM with inert excipients such as starch and dextrin during the evaporation procedure of the herbal extract. These formula granules are produced by various manufacturers, and their qualities may be different in the amount of bioactive compounds. Their quality should be assessed and controlled to assure their therapeutic effects. Huang et al. [73] analyzed hundreds of formula granules made by different manufacturers. Their infrared spectra were compared with those generated from extracts of raw materials. Generally, the contents of bioactive components in formula granules were greater than those in raw materials. Formula granules made from different CMM sources or by various manufacturers could be discriminated by infrared spectra. The similarities and differences among different batches of formula granules manufactured by the same herbal industry could be assessed. Zhou and Tang $[74,75]$ investigated the types and contents of excipients added to formula granules by infrared spectra. They observed that dextrin and lactose were common ingredients and that mixtures of different types of excipients were also used. It was observed that the contents of excipients in formula granules generally varied among manufacturers.

Wu and coworkers [76] analyzed formula granules of Salviae Miltiorrhizae Radix et Rhizoma made by nine different manufacturers by comparing the infrared spectra of the herbal extract, dextrin, and starch. It was found that two formula granules contained a very small amount of excipients, four contained some dextrin, two contained a high content of dextrin, and one contained a high amount of starch ( $\bullet$ Fig. 6). The correlation coefficients of infrared spectra among each formula granule to the reference could give the quantitative evaluation for their similarity.

\section{Quality Control of TCM Injections and Preparations \\ $\nabla$}

The normal and expired 'Qing Kai Ling' injections were identified by infrared and 2D-IR spectra, as well as the mechanism of the deteriorative processes $[77,78]$. The differences between the 2D-IR spectra of the normal and expired 'Qing Kai Ling' injections suggested that its degradation mainly resulted from the oxidation of flavones and the decomposition of glycosides [78]. Zhou et al. also discriminated 'Qing Kai Ling' injections collected from different manufacturers by infrared and 2D-IR spectra [79]. Chen et al. differentiated three types of TCM injections, and all of them were found to contain the extracts of Ginseng Radix et Rhizoma [80].Zhang et al. analyzed the similarities and differences between two injections made from Chrysanthemi Indici Flos (the capitulum of Chrysanthemum indicum L.) and Carthami Flos (the flower of Carthamus tinctorius L.) by infrared and 2D-IR spectra [81]. Yan and coworkers [82] established calibration models by applying ATR techniques and a PLS algorithm to quantify the contents of baicalin and chlorogenic acid in 'Shuang Huang Lian' injections. The determination coefficients $\left(\mathrm{R}^{2}\right)$ of calibration models were 

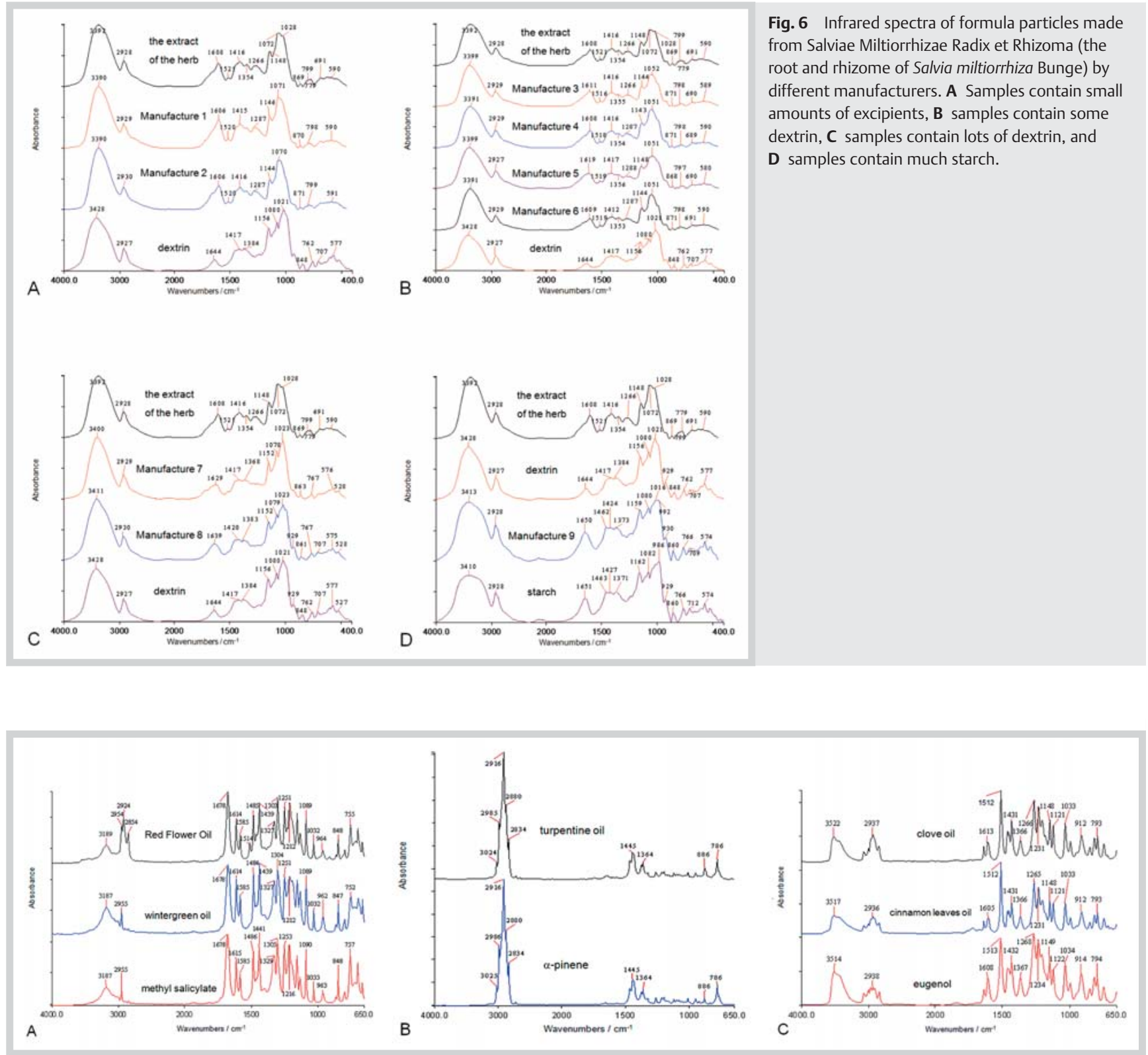

Fig. 7 Infrared spectra of A methyl salicylate, wintergreen oil, and “Red Flower Oil”, B $\alpha$-pinene and turpentine oil, $\mathbf{C}$ eugenol, clove oil, and cinnamon leaves oil.

over 0.99 . The average relative deviations between the predicted contents of the two compounds by infrared spectroscopy models and the amount measured by HPLC were less than $4 \%$. This result indicates that infrared spectroscopy could be a rapid method for the quality control of TCM injections.

'Red Flower Oil', a widely used TCM preparation, is a mixture of several essential oils consisting of wintergreen oil, turpentine oil, clove oil, and cinnamon leaves oil. Wu et al. [83] observed that infrared spectroscopy could be used to identify methyl salicylate as the main compound in wintergreen oil ( $\bullet$ Fig. 7 A), $\alpha$-pinene in turpentine oil ( Fig. 7B), and eugenol in clove oil and cinnamon leaves oil ( Fig. 7C). These 'Red Flower Oil' samples collected from different manufacturers could be discriminated by infrared and 2D-IR spectra. The same author [84] also established calibration models by ATR spectrum and a PLS algorithm to quantitatively analyze methyl salicylate, $\alpha$-pinene, and eugenol in different samples. All determination coefficients $\left(R^{2}\right)$ of calibration models were more than 0.99 for the three compounds. Their values predicted by the infrared spectroscopy models were consistent with those measured by GC.

\section{Conclusions}

$\nabla$

The practice and use of TCM is not only popular in China and some Asian regions, it is also finding appreciation worldwide [85]. As TCM is a multi-composition remedy, its quality is difficult to effectively assess, control, and assure so as to provide the therapeutic actions that the TCM practitioner expects the patient will receive. Fingerprinting is accepted as one of the approaches for quality control of TCM products using analytical techniques such as chromatography, electrophoresis, or spectroscopy pattern-recognition in research publications. Most of these techniques involve "invasive" extraction procedures and do not reflect the 
"true" chemical characteristics of the CMM. Mid-infrared and 2D-IR spectroscopy, which do not require an invasive or extensive sample preparation procedure, combined with appropriate chemometric techniques has been shown to be a useful, rapid, additional, or alternative approach for quality control of CMM and PCM used in TCM treatment.

The main advantages of mid-infrared spectroscopy for the quality control of TCM products are as follows. Firstly, an infrared spectrum provides a "holistic" spectroscopic fingerprinting of all compositions in a tested TCM sample. The variation of both bioactive compounds and unwanted ingredients in tested samples can be shown in the holistic spectroscopic fingerprint thus helping to differentiate and identify good quality from poor quality CMM. Secondly, the operation procedure for sample testing by infrared spectroscopy is simple and rapid. Most CMM samples and PCM products can be directly tested without any extraction, separation, or other preparation. Therefore, chemical composition in tested samples is considered as non-changed, non-damaged. With the availability of software integrating databases, pattern recognition, and calibration models, the quality control of TCM products can be rapidly completed. Currently mid-infrared procedure has been applied to monitor the production of pharmaceutical dosage forms as good manufacturing practice (GMP) in the pharmaceutical industry. With the advancement of modern and database handling technology such an application may be possible in GMP of CMM processing and PCM products in herbal industry. Furthermore, the identity and contents of some chemical compounds in CMM samples can be obtained from infrared spectra by applying some calibration models. Therefore, it is promising and encouraging that mid-infrared spectroscopy offers a rapid alternative or an additional analytical approach for the quality control of TCM products, particularly useful for herbal manufacturers to upgrade the GMP procedure.

\section{Acknowledgements

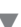

This work was sponsored by the State Administration of Traditional Chinese Medicine of China (2001ZDZX01), the grant of Ministry of Science and Technology of China (2002BA906A29-4), and a Sichuan Provincial grant in China (04SG011-035-1, 2009FZ0053).

\section{Affiliations}

${ }^{1}$ Key Laboratory of the Ministry of Education in China on Bioorganic Phosphorus Chemistry \& Chemical Biology, Department of Chemistry, Tsinghua University, Beijing, China

${ }^{2}$ Key Laboratory of the Ministry of Education in China on the Standardization of Chinese Materia Medica, School of Pharmacy, Chengdu University of Traditional Chinese Medicine, Chengdu, China

${ }^{3}$ Herbal Medicine Research \& Education Centre, Faculty of Pharmacy, University of Sydney and Centre for Complementary Medicine Research, College of Health \& Science, University of Western Sydney, Sydney, Australia

\section{References}

1 Liang YZ, Xie PS, Chan K. Quality control of herbal medicines. J Chromatogr B 2004; 812: 53-70

2 Sun SQ Zhang X, Qin Z, Hu XY. Direct determination of raw plant drugs by FTIR. Spectrosc Spectral Anal 1999; 19: 542-545

3 Sun SQ YuJY, Hu XY. New researches in the direct determination of Chinese traditional drugs with molecular spectroscopy. Spectrosc Spectral Anal 1999; 19: 841-843

4 Sun SQ Zhou Q Yu JY, Hu XY. The latest development of the research on Chinese medicine by molecular vibrational spectroscopy. Spectrosc Spectral Anal 2000; 20: 199-202
5 Liu H, Sun SQ. Application and progress of molecular vibrational spectrum in traditional Chinese medicine modernization. Med Instrum 2005; 5: 6-10

6 Sun SQ, Zhou Q Qin Z. Atlas of two-dimensional correlation infrared spectroscopy for traditional Chinese medicine identification. Beijing: Chemical Industry Press; 2003

7 Noda I. Two-dimensional infrared spectroscopy of synthetic and biopolymers. Bull Am Phys Soc 1986; 31: 520

8 Noda I. Generalized two-dimensional infrared spectroscopy. J Am Chem Soc 1989; 111: 8116-8118

9 Noda I. Generalized two-dimensional correlation method applicable to infrared, Raman, and other types of spectroscopy. Appl Spectrosc 1993; 47: 1329-1336

10 Li YM, Sun SQ Zhou Q Tao JX, Noda I. Study of traditional Chinese animal drugs using FT-IR and 2D-IR correlation spectroscopy. Spectrochim Acta A Mol Biomol Spectrosc 2006; 63: 565-573

11 Wang LQ Li YM, Zhang LP, Xiao HX, Zhou Q Sun SQ. Analysis and characterization of fingerprint infrared spectra for ecology-planted Coptis chinensis. Spectrosc Spectral Anal 2006; 26: 1061-1066

12 Pei LK, Sun SQ Guo BL, Huang WH, Xiao PG. Fast quality control of Herba Epimedii by using Fourier transform infrared spectroscopy. Spectrochim Acta A Mol Biomol Spectrosc 2008; 70: 258-264

13 Cheung YM, Zhou Q Guo BL, Sun SQ. Identifying the characteristics of FTIR spectra of Herba Epimedii Icariin via wavelet analysis and RBF neural network. Spectrosc Spectral Anal 2009; 29: 1830-1834

14 Cao F, Zhou Q Sun SQ. Study on the identification of standard and false Tianma by two-dimensional infrared correlation spectroscopy. Med Instrum 2002; 4: 19-21

15 Zhou Q Li J, Liu J, Huang H, Sun SQ. Two-dimensional correlation infrared spectroscopy of standard and false Dahuang. Chin J Anal Chem 2003; 31: 1058-1061

16 Sun SQ Zhou Q Liu J, Huang H. Study on the identification of standard and false Banxia by two-dimensional infrared correlation spectroscopy. Spectrosc Spectral Anal 2004; 24: 427-430

$17 \mathrm{Xu} \mathrm{CH}$, Zhou Q Sun SQ Wang BQ. The identification of Ejiao by two dimensional correlation infrared spectroscopy. Chin J Anal Chem 2005; 33: 221-224

18 Ayiguli T, Zhou Q Dong XO, Sun SQ. Study on the identification of standard and false Gancao by Fourier transform infrared spectroscopy. Spectrosc Spectral Anal 2006; 26: 1238-1241

19 Zhou J, Sun JY, Xu SY, Zhou Q Sun SQ. Study on the identification of Illicium vatum Hook. f. and Illicium lanceolatum A. C. Smith by multi-steps infrared macro-fingerprint method. Spectrosc Spectral Anal 2008; 28: 2864-2867

20 Huang DL, Sun SQ Xu YQ Chen XK. Analysis and identification of Dangshen and its counterfeit Yeguanmen by Fourier transform infrared spectroscopy. Med Instrum 2008; 5: 22-25

21 Cai F, Sun SQ Yan WR, Niu SJ, Li XE. Identification and analysis of genuine and false Flos Rosae Rugosae by FTIR and 2D correlation IR spectroscopy. Spectrosc Spectral Anal 2009; 29: 2429-2433

22 Chen J, Sun SQ Xu R, Liu YG, Yu J, Liu TN, Li JQ. Identification of Cistanche deserticola from Boschniakla rossica and Cynomorium songaricum using FTIR and two-dimensional correlation IR spectroscopy. Spectrosc Spectral Anal 2009; 29: 1502-1507

23 Yang $P$, Song $P$, Sun SQ Zhou Q Feng S, Tao JX. Differentiation and quality estimation of Cordyceps with infrared spectroscopy. Spectrochim Acta A Mol Biomol Spectrosc 2009; 74: 983-990

24 Cheng ZF, Xu R, Cheng CG. Study on identification of Gastrodia elata BI. by Fourier self-deconvolution infrared spectroscopy. Spectrosc Spectral Anal 2007; 27: 1719-1722

25 Cheng CG, Ying TK. Quality control of Corydalis yanhusuo W. T. Wang by second derivative FTIR spectroscopy combined with statistics. Spectrosc Spectral Anal 2005; 25: 36-38

26 Cheng CG, Sun CR, Pan YJ. Direct identification of Ophiogon japonicus (Thunb.)Ker-Gawl. from its confusable varieties by second derivative FTIR spectroscopy combined with statistics. Spectrosc Spectral Anal 2004; 24: 1055-1059

27 Jin $W Y$, Cheng CG, Wu XH. Identification of Rhizoma Atractylodes based on FTIR spectra and radial basis function network. Spectrosc Spectral Anal 2006; 26: 2210-2213

28 Ma SM, Liu SD, Zhang ZY, Fan GQ. Radial basis function networks and IR spectrometry applied for identification of official Rhubarb samples. Spectrosc Spectral Anal 2005; 25: 874-877 
29 Han MX, Zhou Q Li QH, Sun SQ. Identification of three-step IR spectra of Radix Puerariae from different habitats. Spectrosc Spectral Anal 2009; 29: $1851-1855$

30 Hua R, Sun SQ, Zhou Q Noda I, Wang BQ. Discrimination of Fritillary according to geographical origin with Fourier transform infrared spectroscopy and two-dimensional correlation IR spectroscopy. J Pharm Biomed Anal 2003; 33: 199-209

31 Li YM, Sun SQ Zhou Q Qin Z, Tao JX, Wang J, Fang X. Identification of American ginseng from different regions using FT-IR and two-dimensional correlation IR spectroscopy. Vib Spectrosc 2004; 36: 227-232

32 Zhou X, Sun SQ Huang QH. Identification of Pericarpium Citri Reticulatae from different regions using FTIR. Spectrosc Spectral Anal 2007; 27: 2453-2455

33 Sun SQ Yuan ZM, Bai Y. Identify the genuine of Shanyao (Dioscorea opposite Thunb.) by FTIR combined with computer aided analysis. Comput Appl Chem 2002; 19: 77-80

$34 X u Y Q$ Sun SQ Yuan ZM, Bai Y. Discrimination of trueborn tuber dioscoreae by fingerprint infrared spectra and principal component analysis. Chin J Anal Chem 2002; 30: 1231-1233

35 Sun SQ TangJM, Yuan ZM, Bai Y. FTIR and classification study on trueborn tuber dioscoreae samples. Spectrosc Spectral Anal 2003; 23: 258-261

36 Zhou Q, Sun SQ Leung HW. FTIR and classification study on Gouqi from different cultural areas. Spectrosc Spectral Anal 2003; 23: 509-511

37 Liu SH, Zhang XG, Zhou Q Sun SQ. Use of FTIR and pattern recognition to determine geographical origins of Chinese medical herbs. Spectrosc Spectral Anal 2005; 25: 878-881

38 Xu YQ Sun SQ, Feng XF, Hu SL. Quick identification of Skullcaps in different geographical origins using clustering analysis method and infrared fingerprint spectra. Spectrosc Spectral Anal 2003; 23: 502-505

39 Xu YQ Huang H, Zhou Q Zhou HT, Hu SL, Sun SQ. The application of fingerprint infrared spectra and clustering analysis in the discrimination of geographical origin of Paeonia lactiflora Pall. Chin J Anal Chem 2003; 31: 5-9

40 Wang FL, Zhou JK, Wu J, Yu L, Sun SQ. Study on the identification of cultivated and wilding Danshen by multi-steps infrared macro-fingerprint method. Med Instrum 2006; 5: 18-20

41 Liu D, Li YG, Xu H, Sun SQ Wang ZT. Differentiation of the root of Cultivated Ginseng, Mountain Cultivated Ginseng and Mountain Wild Ginseng using FT-IR and two-dimensional correlation IR spectroscopy. J Mol Struct 2008; 883: 228-235

42 Liu G, Dong Q Yu F, Liu JH, Sun SZ. Identification of Gastrodia elata Blume by Fourier transform infrared spectroscopy. Spectrosc Spectral Anal 2004; 24: 308-310

43 Dong B, Sun SO, Zhou HT, Hu SL. Rapid and undamaged determination of Chishao by Fourier-transform infrared spectroscopy and clustering analysis. Spectrosc Spectral Anal 2002; 22: 232-234

44 Dong B, Sun SQ Qin Z, Zhou HT, Hu SL. Rapid and undamaged determination of Chishao by Fourier-transform infrared spectroscopy and clustering analysis. Comput Appl Chem 2002; 19: 89-92

45 Xu R, Sun SQ Liu YG, Yu J, Zhou F, Liu TN, Chen J, Chen SL. Rapid and undamaged determination of Cistanche deserticola by Fourier-transform infrared spectroscopy and clustering analysis. Spectrosc Spectral Anal 2009; 29: 1860-1863

$46 \mathrm{Xu}$ YQ Zhou Q Sun SQ Cai SQ. Recognition of three classes of Skullcaps by FTIR spectroscopy combined with artificial neural networks. Spectrosc Spectral Anal 2002; 22: 945-948

47 Huang $H$, Sun SQ Xu JW, Wang Z. Novel application of FTIR in medical herb chemotaxonomy. Spectrosc Spectral Anal 2003; 23: 253-257

$48 \mathrm{Lu} \mathrm{GH}$, Zhou Q Sun SQ Leung KSY, Zhang H, Zhao ZZ. Differentiation of Asian ginseng, American ginseng and Notoginseng by Fourier transform infrared spectroscopy combined with two-dimensional correlation infrared spectroscopy. J Mol Struct 2008; 883: 91-98

49 Wang Z, Sun SQ Li XB, Zhou Q Lin L, Du DG. Direct determination of Rhizoma Cimicifuga by FTIR spectroscopy. Spectrosc Spectral Anal 2001; 21: 311-313

50 Peng Y, Sun SQ Zhao ZZ, Leung HW. A rapid method for identification of genus Lycium by FTIR spectroscopy. Spectrosc Spectral Anal 2004; 24: 679-681

51 Pei $L K$, Guo BL, Sun SQ Huang WH. Study on the identification of some species of Herba Epimedii with FTIR. Spectrosc Spectral Anal 2008; 28: 55-60

52 Chen JB, Zhou Q Noda I, Sun SQ. Quantitative classification of two-dimensional correlation spectra. Appl Spectrosc 2009; 63: 920-925
53 Chen JB, Zhou Q Noda I, Sun SQ. Discrimination of different genera Astragalus samples via quantitative symmetry analysis of two-dimensional hetero correlation spectra. Anal Chim Acta 2009; 649: 106-110

$54 \mathrm{Lu} \mathrm{GH}$, Sun SQ Leung HW, Chan KKC. Study on the identification of Dangguitou and Dangguiwei by two-dimensional infrared correlation spectroscopy. Spectrosc Spectral Anal 2004; 24: 311-314

55 Jin ZX, Xu SY, Sun SQ, Zhou Q. Analysis of Acanthopanax senticosus Harms for different parts using Fourier transform infrared spectroscopy. Spectrosc Spectral Anal 2008; 28: 2859-2863

$56 \mathrm{Xu}$ R, Sun SQ Liu YG, Chen J, Chen SL, Zhou F. Analysis and evaluation of different radial part of Cistanche deserticola by Fourier transform infrared spectroscopy and two-dimensional infrared correlation spectroscopy. Chin J Anal Chem 2009; 37: 221-226

57 Hong QH, Cheng ZF, Cheng CG. Application of FTIR spectroscopy to the analysis of quality mensuration of Paeonia lactiflora Pall. from native habitat. Spectrosc Spectral Anal 2006; 26: 1610-1613

58 Zhan DQ Cheung YM, Sun SQ. Wavelet-transform based identification of ginseng of different ages using two-dimensional infrared correlation spectroscopy. Spectrosc Spectral Anal 2007; 27: 1497-1501

59 Zhou X, Sun SQ Huang QH. Identification of Pericarpium Citri Reticulatae from different years using FTIR. Spectrosc Spectral Anal 2008; 28: 72-74

60 Sun SQ Wang MQ Leung HW, Yeung HW. A rapid method for the identification of four different features of Gouqizi by FTIR spectrometry. Spectrosc Spectral Anal 2001; 21: 787-789

61 Liu Y, Wang JQ Liu SH, Sun SQ. Two-dimensional correlation infrared spectroscopy applied to analyzing and identifying the Radix Paeoniae Alba medicinal materials. J Mol Struct 2008; 883: 137-141

62 Yu L, Sun SQ Fan KF, Zhou Q Noda I. Research on processing medicinal herbs with multi-steps infrared macro-fingerprint method. Spectrochim Acta A Mol Biomol Spectrosc 2005; 62: 22-29

$63 \mathrm{Yu}$ L, Sun SQ Zhou Q Qin Z. Research on parching procedure of white mustard seed with Fourier transform infrared spectroscopy and twodimensional IR correlation spectroscopy. Spectrosc Spectral Anal 2006; 26: 2181-2185

64 Tang JM, Xian D, Sun SQ. Study on Radix Aconiti and Radix Aconiti Preparata by two-dimensional infrared correlation spectroscopy. Med Instrum 2005; 3: 27-30

65 Wang CL, Wen JM, Cheng T, Jiang KW, Sun SQ Tu Y. Study on Aconitum kusnezoffii Reichb. and its processed products by 2D-IR correlation spectroscopy. Spectrosc Spectral Anal 2009; 29: 1498-1501

66 Sun SQ Zhou O Leung HW. Study on Paofupian, Heishunpian and Baifupian by two-dimensional infrared correlation spectroscopy. Spectrosc Spectral Anal 2003; 23: 1082-1085

67 Bao HJ, An H, Bai Y, Sun SQ. The analysis and identification of Chrysanthemum and processed Chrysanthemum by IR and second-order derivative spectroscopy. Med Instrum 2004; 5: 26-28

$68 \mathrm{Xu} C \mathrm{CH}$, Sun SQ Guo CQ Zhou Q Tao JX, Noda I. Multi-steps infrared macro-fingerprint analysis for thermal processing of Fructus Viticis. Vib Spectrosc 2006; 41: 118-125

69 Liu HX, Sun SQ Lv GH, Chan KKC. Study on Angelica and its different extracts by Fourier transform infrared spectroscopy and two-dimensional correlation IR spectroscopy. Spectrochim Acta A Mol Biomol Spectrosc 2006; 64: 321-326

70 Liu HX, Sun SQ Lv GH, Liang XY. Discrimination of extracted lipophilic constituents of Angelica with multi-steps infrared macro-fingerprint method. Vib Spectrosc 2006; 40: 202-208

71 Liu HX, Zhou Q Sun SQ Bao HJ. Discrimination of different Chrysanthemum with Fourier transform infrared spectroscopy. J Mol Struct 2008; 883: $38-47$

72 Wu YW, Xiao XH, Sun SQ Liu HX. Study on traditional Chinese medicine extracts of Rhizoma Coptidis by FTIR spectroscopy. Spectrosc Spectral Anal 2009; 29: 93-96

73 Huang H, Jing L, Qin Z, Zhou Q Sun SQ. The application of Fourier transform infrared spectroscopy on the quality control of traditional Chinese medicine formula particles. Chin J Anal Chem 2003; 31: 828-832

74 Zhou Q Li J, Sun SQ Liang XY. Rapid identification of traditional Chinese medicine formula particles by Fourier transform infrared spectroscopy. Chin J Anal Chem 2003; 31: 292-295

75 Tang JM, Sun SQ Yuan ZM, Qin Z. Study on the rapid and nondestructive identification of TCM formula particles by FTIR. Spectrosc Spectral Anal 2004; 24: 554-556

$76 \mathrm{Wu}$ J, Sun SQ Zhou Q Yu L. Study on rapid and nondestructive analysis of Danshen formula particles by FTIR. Spectrosc Spectral Anal 2007; 27: $1535-1538$ 
77 Zuo L, Sun SQ Tang JM. Two-dimensional correlation analysis of FT-IR spectra for research on the thermal stability of the Qingkailing injection. Comput Appl Chem 2002; 19: 95-98

78 Zuo L, Sun SQ Zhou Q Tao JX, Noda I. 2D-IR correlation analysis of deteriorative process of traditional Chinese medicine 'Qing Kai Ling' injection. J Pharm Biomed Anal 2003; 30: 1491-1498

79 Zhou Q Sun SQ Zuo L. Study on traditional Chinese medicine 'Qing Kai Ling' injections from different manufactures by 2D IR correlation spectroscopy. Vib Spectrosc 2004; 36: 207-212

80 Chen JB, Zhou Q Sun SQ Yu L, Xu KY. Study on quality control of traditional Chinese medicine ginseng injection with Fourier transform infrared spectroscopy. Spectrosc Spectral Anal 2007; 27: 1493-1496

81 Zhang P, Sun SQ Zhou Q Xu KY. Study on quality control of safflower injection and wild chrysanthemum injection with Fourier transform infrared spectroscopy. Spectrosc Spectral Anal 2007; 27: 1539-1542
82 Yan S, Xu ML, Tu Y, Li HF, Sun SQ. Qualitative and quantitative analysis of Shuanghuanglian fenzhenji by FTIR. Spectrosc Spectral Anal 2009; 29: 1558-1561

83 Wu YW, Sun SQ Zhou Q Tao JX, Noda I. Volatility-dependent 2D IR correlation analysis of traditional Chinese medicine 'Red Flower Oil' preparation from different manufacturers. J Mol Struct 2008; 882: 107-115

$84 \mathrm{Wu} Y W$, Sun SQ Zhou Q Leung HW. Fourier transform mid-infrared (MIR) and near-infrared (NIR) spectroscopy for rapid quality assessment of Chinese medicine preparation Honghua Oil. J Pharm Biomed Anal 2008; 46: 498-504

85 Chan $K$. Chinese medicinal materials and their interface with Western medical concepts. J Ethnopharmacol 2005; 96: 1-18 